\title{
Direction of Arrival Estimation in Multiple Antenna Arrays by Using Power Delay Profile for Random Access Performance in 5G Networks
}

\author{
O. AYDIN and T. AKYÜZ
}

\begin{abstract}
In the transition from 4G to 5G, various solutions are being developed to improve performance for features such as data rates, latency, connectivity density and reliability. One of these features is Random Access Procedure because of the increasing number of users in 5G networks. In the random access method, user equipment performs a random access to the base station with a preamble and thus registers itself to the base station. However, if more than one user equipment use the same preamble at the same time, collision occurs and the registration process in the base station could be halted. In this paper, a new method is proposed which can be used to calculate Direction of Arrival between adjacent antenna signals in the antenna array with the help of the phase differences. Thus, the collision can be avoided by using the beamforming technique of the MIMO system using the calculated arrival angle of the user equipment. The proposed method is verified for two and three user equipment placed at different angles, at different distances to an antenna array consisting of 10 antennas.
\end{abstract}

Index Terms-Direction of Arrival (DOA), Power Delay Profile, Preamble, Zadoff-Chu.

\section{INTRODUCTION}

$I^{2}$ T IS EXPECTED that in the Fifth Generation (5G) wireless communication systems, the connection density will increase more than tenfold compared to the Fourth Generation (4G) systems [1]. As a result of this increase, it is clear that user elements (UE) will cause significant congestion in accessing LTE services. To meet this demand, one of the solutions is the using location division multiplexing systems which is supported with multi input multi output (MIMO) antenna systems in addition to time and frequency division

OMER AYDIN, is with the Department of Research and Development, Netas, 34912, Turkey,(e-mail: aydin@ netas.com.tr). Also with Department of Electrical and Electronics Engineering, Istanbul Technical University, Istanbul, Turkey.

iD https://orcid.org/0000-0002-1519-6937

TUĞRUL AKYÜZ, is with the Department of Research and Development, Netas, 34912, Turkey (e-mail: tugrulvega@gmail.com).

iD https://orcid.org/0000-0001-8297-8720

Manuscript received March 8, 2019; accepted May 25, 2019. DOI: $10.17694 /$ bajece. 537262 multiplexing structures with the intelligent deployment of Resource Elements (RE) [1]. Furthermore, Random Access (RA) that UEs use for the first access to base station is one of the bottlenecks that make LTE services difficult to access and the number of preambles, already used in $4 \mathrm{G}$ networks to solve the Random Access collision problem seems to be insufficient. It is clear that solutions that solve this congestion will enable more UEs to be registered to the base station more quickly.

Registration of the UE to the base station is done over the Random Access Channel (RACH) in Long Term Evolution (LTE) networks. However, concurrent access of UEs to the same channel causes signal collision. To overcome this problem, a preamble structure was introduced in $4 \mathrm{G}$. With the help of the preamble, the randomly selected Zadoff Chu sequences are being used to eliminate the effects of collisions at the base station [2], [3].

Since the number of users in $5 \mathrm{G}$ networks is expected to be much more than the $4 \mathrm{G}$ network, the number of Zadoff Chu series may be insufficient for the first registration of the UEs. Reusing the same Zadoff Chu series on the random access channels which are multiplexed in the 3D space with the help of MIMO antenna system might be a solution to the this problem. To do this efficiently, it is very important to correctly calculate the Direction of Arrival (DoA) of the different UEs in the coverage area of the base station. Using angular position information, the initial registration and channel allocation procedures for UEs can be performed using the same Zadoff Chu series with the help of the RACH Procedure.

DoA resolution algorithms have been extensively investigated and in a variety of solutions were proposed in the literature. Delay and Collection techniques such as Bartlett method tries to magnify the signals from certain direction by compensating the phase shift [4], [5].The Capon method is a minimum variance method that estimates direction of arrival by changing weight to minimize the array power subject to unity gain [6]. MUSIC (MUltiple SIgnal Classification) and ESPRIT (Estimation of Signal Parameters via Rotational Invariance Technique) algorithm, which are based on the subspace method, are developed based on eigenvectors, eigenvalues and spectral matrix theories [7]. The above algorithms use the correlation of the signals received by the 
different antennas in the multi-antenna structure. Apart from these studies, subcarrier-based beamforming methods and multi-level beamforming approaches for more efficient operation are available [8]. Some studies also use two dimensional antenna arrays [9]. Other recent studies on DOA estimation are using MUSIC algorithm after rejecting interferences [10], wideband DOA estimation by using adaptive array technique [11], DOA estimation in cyclic prefix OFDM systems by using mono pulse ratio [12]. The estimation of the reception angle is proposed in [13] based on the distribution characteristics using power delay profile.

The above-mentioned methods evaluate the correlation of all incoming signals; therefore, they also take into account the signals of the UEs having different input preamble. These algorithms have an upper limit on the number of source UEs to be calculated depending on the number of antennas used in the array antenna structure of the base station [9]. Therefore, the use of these algorithms at the receiver input gives the main difficulty in accurately determining the desired arrival angle of a large number of UEs.

In this paper, a new method is proposed to overcome the above-mentioned problem, which is based on using the phase differences of the peak values in the Power Delay Profile array, which is already calculated in the receiver stage of OFDM layer, used LTE systems [1], [2].

\section{PROPOSED METHOD}

\section{A. Preamble and Zadoff Chu Sequence}

The RACH is the channel (Random Access Channel) for mobile users to access the base station. Physical structure of the RACH, Physical Random Access Channel (PRACH) is shown in the Fig. 1.

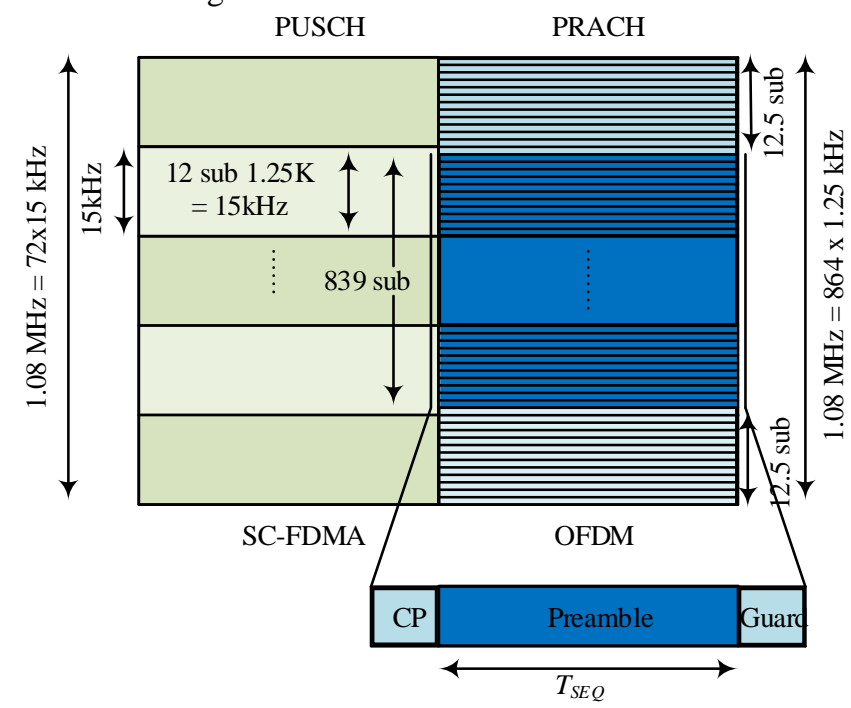

Fig.1. Structure of the Physical Random Access Channel (PRACH)

Access through this channel continues with the mutual acknowledgment mechanisms and the mobile device receives the configuration of the resource blocks (RB) that it needs from the base station. The Random Access of the UEs to the base station is achieved by preamble signatures produced using Zadoff $\mathrm{Chu}$ arrays which are perpendicular to each other, that provides the detection of UEs by the base station.

The Zadoff Chu sequence $z_{u}$ is obtained from the following formula.

$$
z_{u}(n)=e^{\frac{-j \pi u n(n+1)}{N_{Z C}}} n=0,1,2 \ldots . N_{Z C}-1
$$

where $u \in\{1,3,5 \ldots, \mathrm{U}-1\}, \mathrm{U}$ is the root index and $N_{Z C}$ length of the Zadoff Chu sequence [2].

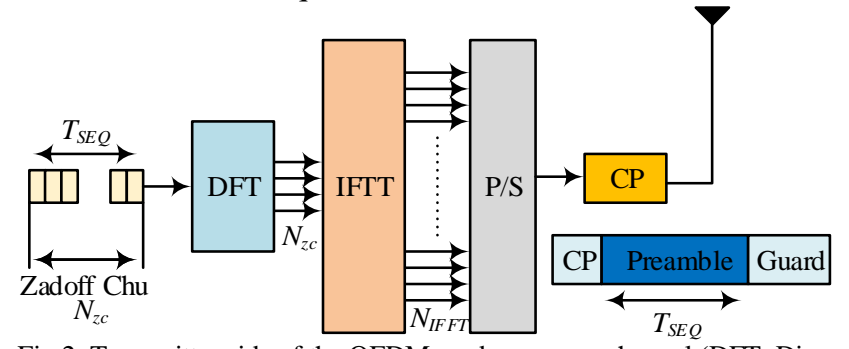

Fig.2. Transmitter side of the OFDM random access channel (DFT: Discrete Fourier Transform, IFFT: Inverse Fourier transform, P/S: parallel/serial, CP: Cyclic Prefix)

As shown in Fig. 2, the user terminal initially selects a Zadoff Chu sequence. Afterwards, Discrete Fourier Transform (DFT) is performed to the Zadoff Chu series which has a sequence length of $N_{z c}$. The array of frequency signals of DFT output is then located at the $1.08 \mathrm{MHz}$ range in the middle region of the Orthogonal Frequency Division Multiplexing (OFDM) band according to carrier frequencies of $1.25 \mathrm{kHz}$ as shown in Fig. 1 [2]. Then, Inverse Fast Fourier Transform (24576 point IFFT) is applied to the carrier frequencies of $1.25 \mathrm{kHz}$ (Fig. 2). The preamble signal of $0.8 \mathrm{~ms}$ long is then converted from the parallel to the serial by adding the cyclic prefix $(\mathrm{CP})$ and guard time, and then sent to the antennas [2]. In the receiver part, as shown in Fig. 3, the inverse of the sending procedure is applied, and the preamble is obtained.

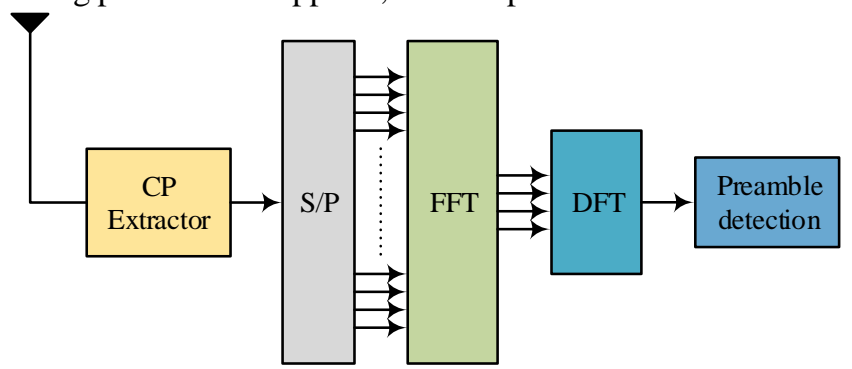

Fig.3. OFDM random access receiver channel

\section{B. Power Delay Profile and DoA}

The signal sent from the UEs received from the PRACH channel is pre-processed and preamble detection is performed according the defined standards as shown in Fig. 4.

Assumed that $x(n)$ is to be the sequence of the signal received, the Power-Delay Profile, $P D P(l)$ is calculated by the following formula:

$P D P(l)=|p(l)|^{2}=\left|\sum_{n}^{N_{z c}-1} x(n) z_{u}^{*}\left[(n+l) \bmod N_{z c}\right]\right|^{2}$

where $z_{u}(n)$ is the reference Zadoff Chu for the root index $u$ and $z_{u}(n)^{*}$ indicates the complex conjugate of $z_{u}(n)$.

As a result of this process which is shown in the block diagram in Fig. 4b, peak values occur only when the Zadoff 
Chu series generated from the root index selected by the corresponding UE to generate the preamble for the same root index. The distance between the peaks of this array to the reference point gives the time of arrival (ToA) of signals sent by the UEs. For example, if there are two UEs, two peaks are formed in the array for each UE as can be seen from Fig. 5. The signal from an UE sometimes reaches to the receiver after being reflected from an obstacle; in this case, a relatively weak peak may be seen with a delay as shown from the figure.

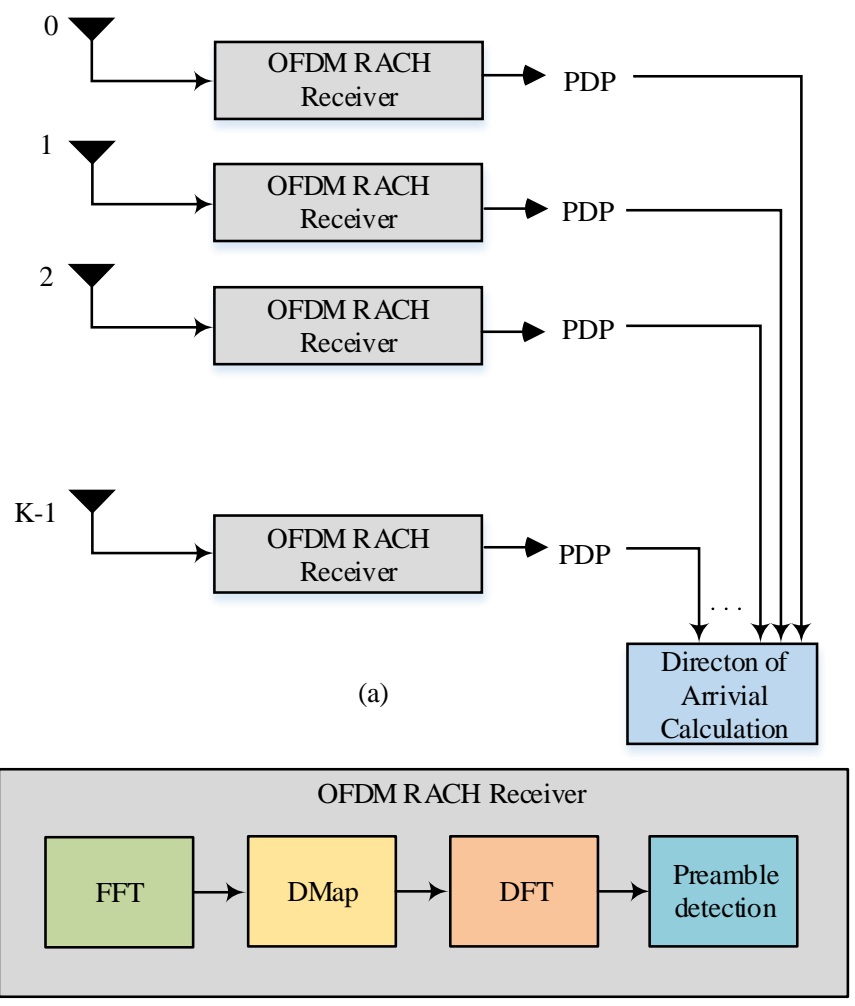

(b)

Fig.4. Block diagram of the receiver part with the antenna array and preprocessor blocks which calculate PDP (a) and sub-blocks of the OFDM $\mathrm{RACH}$ receiver (b)

PDP Amplitude

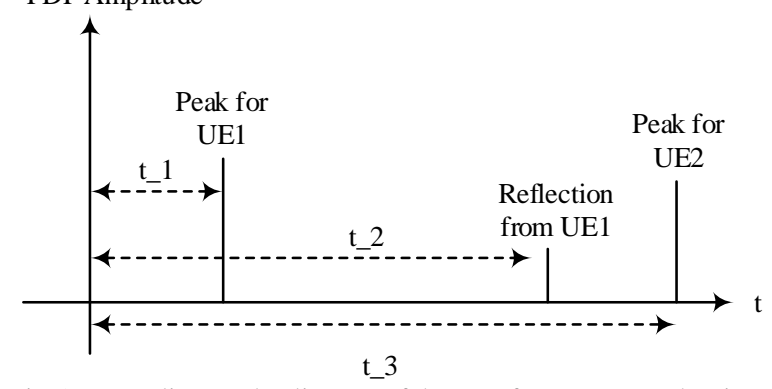

Fig.5. Depending on the distance of the UEs from antenna, the signals reach the receiver at different time, and peaks are formed at these points; the reflected signals form relatively low peaks

In the case of multiple antenna structures, this process is applied on every antenna. The resulting Power Delay Profile sequences can be used for the direction of the arrival detection method. Obtaining the UE's signal input angles, gives the possibility to the base station to continue to perform the RACH procedure differently for each of the mobile terminals by using beam forming with the help of the antenna array.

\section{PHASE DIFFERENCE IN POWER DELAY PROFILE}

The signal from one UE to each antenna will come with a certain phase difference, both depending on the angle of arrival of the signal to the antenna array and the distance between the antennas. As can be seen in Fig. 6, time delays occur in the signals sent by the UEs at a certain angle relative to the range $d$ between the antennas. The constant $c$ defines propagation waves at light speed. $\Delta_{t k}$ is $k$ th antenna signal arriving delay time. Assuming that $s_{b}(t)$ is a narrow base band signal and $f_{c}$ is the carrier frequency, the real part of this signal becomes $s(t)=\operatorname{Re}\left\{s_{b}(t) e^{-j 2 \pi f_{c} t}\right\}$.

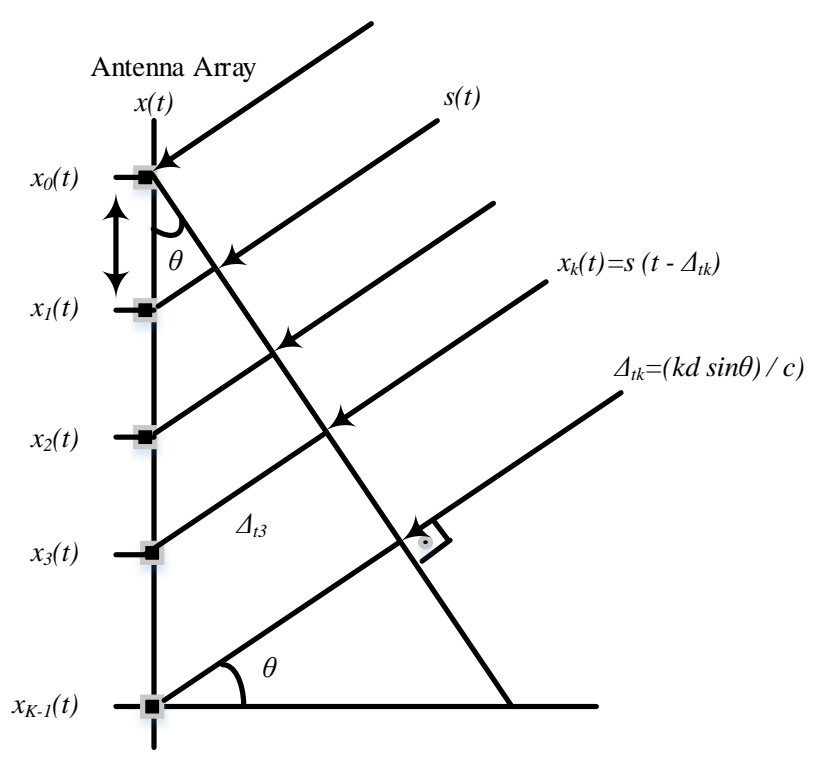

Fig.6. UE signal to the antenna array with the angle of $\theta$

We can write $x_{k}(t)$ at the receiver such that:

$$
\begin{gathered}
x_{k}(t)=\operatorname{Re}\left\{s_{b}\left(t-\Delta_{t k}\right) e^{-j 2 \pi f_{c}\left(t-\Delta_{t k}\right)}\right\} \\
x_{k}(t)=\operatorname{Re}\left\{s_{b}\left(t-\Delta_{t k}\right) e^{-j 2 \pi f_{c} \Delta_{t k}} e^{-j 2 \pi f_{c} t}\right\}
\end{gathered}
$$

and for the base band, we can write

$$
x_{k}(t)=s_{b}\left(t-\Delta_{t k}\right) e^{-j 2 \pi f_{c} \Delta_{t k}}
$$

and then, when we put $n T$ instead of $t$, we get

$$
x_{k}(n T)=s_{b}\left(n T-\Delta_{t k}\right) e^{-j 2 \pi f_{c} \Delta_{t k}}
$$

where $T$ is the sampling period. Assuming that $T \gg \Delta_{t k}$, we get

$$
x_{k}(n T) \cong s_{b}(n T) e^{-j 2 \pi f_{c} \Delta_{t k}}
$$

We have $\Delta_{t k}=k d \sin \theta / c$ and $f_{c} \lambda=c$, so when we select $d=\lambda / 2$ we get;

$$
\begin{gathered}
x_{k}(n T)=s_{b}(n T) e^{-j 2 \pi f_{c} \frac{k d \sin \theta}{c}} \\
x_{k}(n T)=s_{b}(n T) e^{-j \pi k \sin \theta}
\end{gathered}
$$

In discrete domain for $k$ th antenna we can write:

$$
x_{k}[n]=s[n] e^{-j \pi k \sin \theta}
$$

We get $p_{k}(l)$ as below after the cross correlation by substituting Equation (10) into Equation (2): 
BALKAN JOURNAL OF ELECTRICAL \& COMPUTER ENGINEERING, Vol. 7, No. 2, April 2019

$$
p_{k}(l)=e^{-j \pi k \sin \theta} \sum_{n}^{N_{z c}-1} s[n] z_{u}^{*}\left[(n+l) \bmod N_{z c}\right]
$$

In each $p_{k}(l)$ series for each $k$ th antenna there is a phase difference angle of $\pi k \sin \theta$. If we consider the peaks in the $p_{k}(l)$ series that indicate the signal from a UE, there is a phase difference between the $p_{k}\left(l_{\text {peak }}\right)$ of the $k$ th antenna and the $p_{k+1}\left(l_{\text {peak }}\right)$ of the $(k+1)$ th antenna. The phase difference $\phi$ can be calculated as:

$$
\phi=\angle\left(p_{k}\left(l_{\text {peak }}\right) p_{k+1}\left(l_{\text {peak }}\right)\right)
$$

and direction of arrival $\theta$ is

$$
\theta=\sin ^{-1} \frac{\phi}{\pi}
$$

assuming that phase difference between adjacent antennas less than $180^{\circ}$.

\section{Simulation Results}

The simulation was performed in MATLAB using two UEs positioned against to the base station at a distance of $357 \mathrm{~m}$ and $2145 \mathrm{~m}$ with the angle of $30^{\circ}$ and $-10^{\circ}$ degrees to the linear antenna array respectively.

The antenna array consists of $K=10$ antennas spaced at distance $d=\lambda / 2$. As shown in Fig. 7, when the broadcast frequency is selected as $3 \mathrm{GHz}$, this spacing distance $d$ should be about $5 \mathrm{~cm}$.

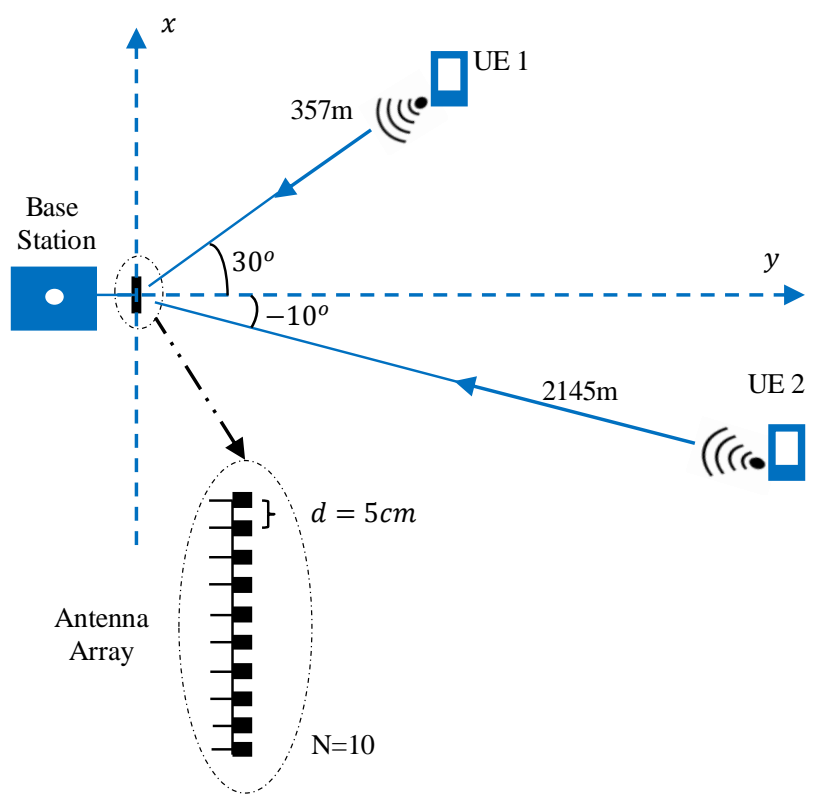

Fig.7. Configuration of the UEs and antenna array to simulate proposed DoA calculation method

The preamble index of the two UEs in RACH channel was chosen as one. As a result, when the same Zadoff Chu sequence is used in the two UEs for random access, in the Power Delay Profile series, two peaks are formed as shown in Fig. 8.

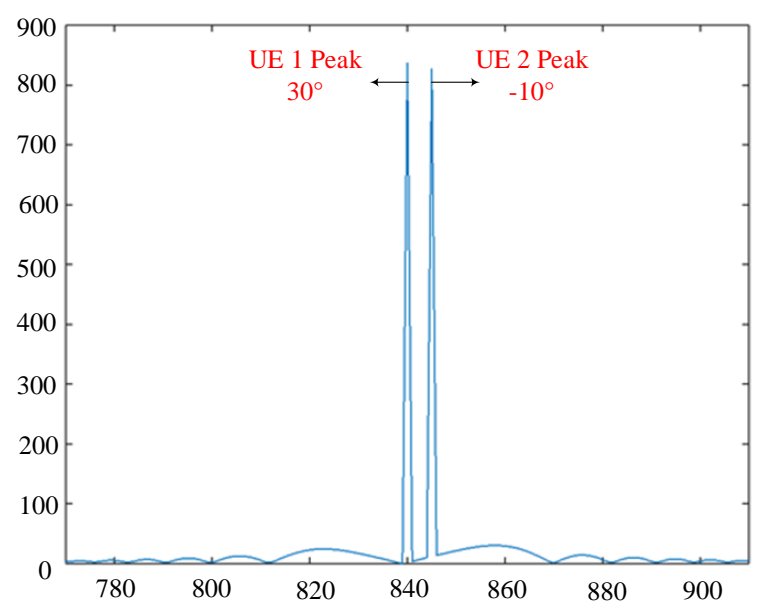

Fig.8. Peak values of the Power Delay Profile, UE 1 and UE 2 obtained as a result of the preamble detection. The horizontal axis shows the sampled data index

To find the direction of arrival, the phase shifts at the peak complex values are used. These phase shifts are shown in Fig. 9 for the fifth and sixth antennas of the antenna array in the base station. As mentioned above, $\theta$ is the direction of arrival to be found, and the phase shift is $\phi=\pi \sin \theta$ as from the Equation (13). Since the phase in each consecutive adjacent antenna is approximately $\theta$, the average of this values increase the accuracy. The average is calculated as follows:

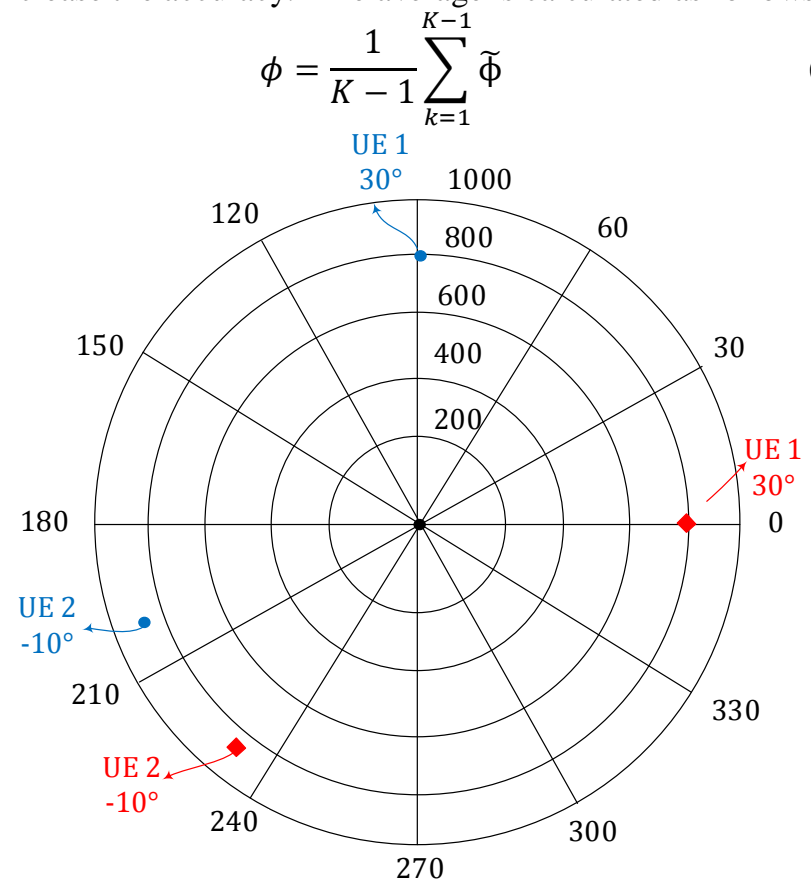

Fig.9. Peak values of Power Delay Profile for the fifth (red rectangular) and sixth (blue circles) antenna view in the polar coordinate

The second simulation is made by adding a third UE with same Zadoff Chu sequence, in addition to the previous two UEs and positioned at an angle of $-20^{\circ}$ at a distance of $3576 \mathrm{~m}$. With this simulation, the effect of the third UE which uses the same Zadoff Chu sequence, on the results obtained in the previous simulation is examined. Peak values of the Power Delay Profile for three UEs are shown in Fig. 10. 


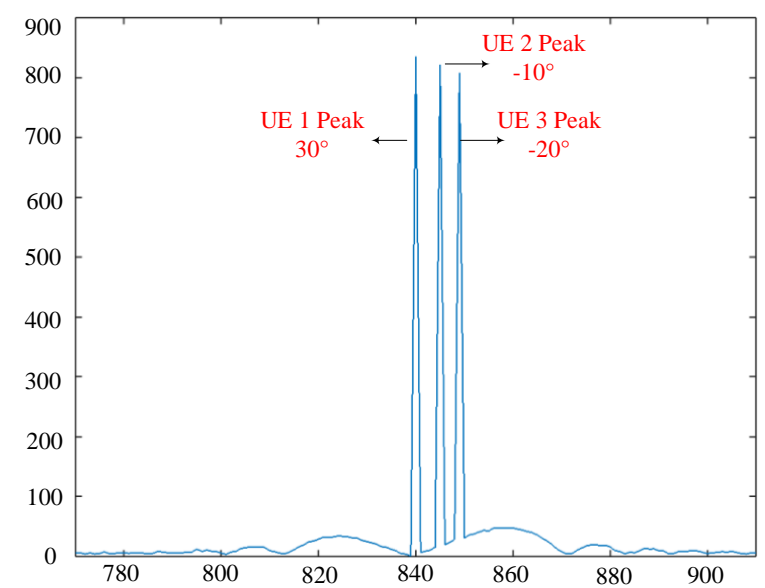

Fig.10. Peak values of the Power Delay Profile for three UEs using same Zadoff Chu sequence.

UE 1

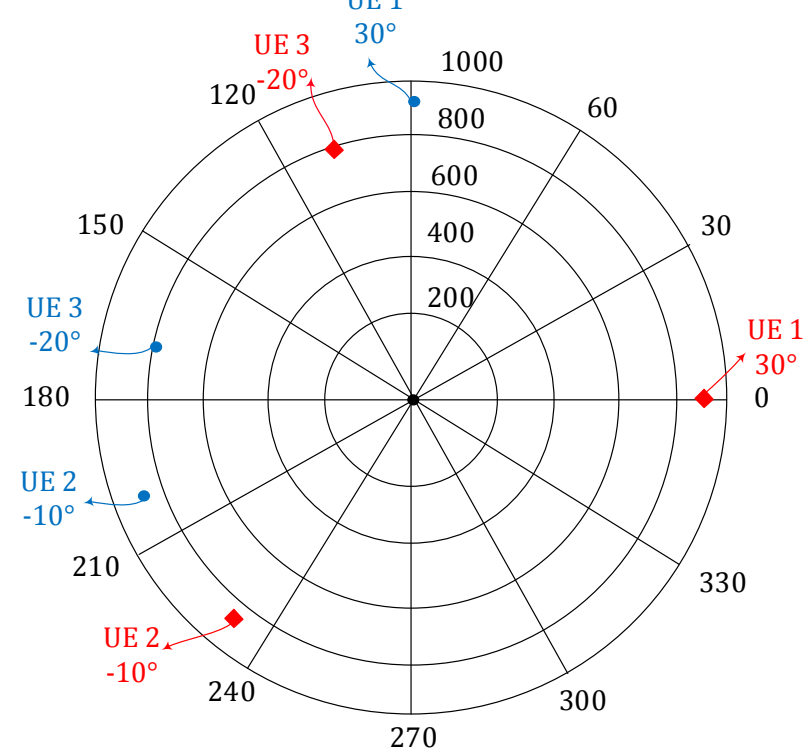

Fig.11. Peak values of Power Delay Profile for the fifth (red rectangular) and sixth (blue circles) antenna for three UEs

As can be seen from Fig. 11, the positions calculated in the first simulation for the first and second UE are not affected by the third UE. The location of the UE 3 is also calculated correctly.

\section{CONCLUSION}

It is expected that the number of users in $5 \mathrm{G}$ networks will be much higher than the number of preambles that can ensure that the UEs are successfully registered to the base station. In this paper, a solution is proposed that allows the simultaneous use of same preambles to increase the registration performance. For this purpose, using the power delay profile, the angle of receiving signal sent by the UE can be calculated to enable beam-forming functionality, which can be performed with the help of the antenna array. The phase difference of the incoming signals from the neighbouring antennas is calculated by using peak values which are already calculated OFDMA layers with the help of Zadoff Chu series in $4 \mathrm{G}$ networks.

The success of the proposed method verified by using MATLAB with an antenna array consisting 10 antennas. Simulations are made for two different configurations, first consists two UEs and the second with third UE additional to the first configuration. All UEs which use same Zadoff Chu sequences in the preamble are located at different distance and angles. Simulation result proves that, the proposed method can be a candidate method for solving the simultaneous registration problem of multiple UEs in $5 \mathrm{G}$ systems.

\section{REFERENCES}

[1] A. Gupta, R. K. Jha, "A Survey of 5G Network: Architecture and Emerging Technologies", IEEE Access, vol. 3, 2015, pp.1206-1232.

[2] $3^{\text {rd }}$ Generation Partnership Project; Technical Specification Group Radio Access Network; Evolved Universal Terrestrial Radio Access (E-UTRA); Physical Channels and Modulation (Release 10), 2011.

[3] H. J. Zepernick, A. Finger, Pseudo Random Signal Processing: Theory and Application, John Wiley \& Sons, 2013.

[4] V. Krishnaveni, T. Kesavamurthy, B. Aparna, "Beamforming for Direction-of-Arrival (DOA) Estimation-A Survey", International Journal of Computer Applications, vol. 61, 11, 2013, pp.4-11.

[5] Y. Khmou, S. Safi, M. Frikel, "Comparative Study between Several Direction Of Arrival Estimation Methods", Journal of Telecommunications and Information Technology, vol. 1, 2014, pp.4148.

[6] R. J. Weber, Y. Huang, "Analysis for Capon and MUSIC DOA estimation algorithms", IEEE Antennas and Propagation Society International Symposium, June 2009.

[7] J. S. Jeong, K. Sakaguchi, J. I. Takada, K. Araki, "Performance of MUSIC and ESPRIT for joint estimation of DOA and angular spread in slow fading environment", IEICE Transactions on Communications, vol. E85-B, 5, 2002.

[8] A. Abdelkader, S. Shahbazpanahi, S. Gershman, "Joint subcarrier power loading and distributed beamforming in OFDM-based asynchronous relay networks", 3rd IEEE International Workshop on Computational Advances in Multi-Sensor Adaptive Processing (CAMSAP), 2009.

[9] S. Sesia, I. Toufik, M. Baker, LTE - The UMTS Long Term Evolution From Theory to Practice, John Wiley \& Sons, 2013.

[10] K. Kataoka, N. Kikuma, K. Sakakibara, "DOA Estimation of Desired Wave with Interference Rejection Using Beamspace Root-MUSIC", International Symposium on Antennas and Propagation (ISAP), 2018.

[11] B. Yin, "Wideband Signal Direction-of-Arrival Estimation by Using Steered Adaptive Array Technique”, IEEE International Conference on Signal Processing, Communications and Computing (ICSPCC), 2018.

[12] H. Kim, J. Kim, K. H. Lee, K. S. Kim, "DOA estimation in Cyclic Prefix OFDM Systems in LOS mmWave Channel using Monopulse Ratio", International Conference on Information and Communication Technology Convergence (ICTC), 2018.

[13] C. ZióBkowski, J. M. Kelner, "Estimation of the Reception Angle Distribution Based on the Power Delay Spectrum or Profile", Hindawi Publishing Corporation International Journal of Antennas and Propagation, vol. 2015.

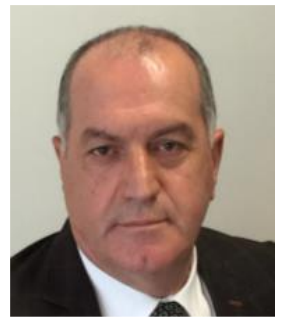

\section{BIOGRAPHIES}

OMER AYDIN has received his B.Sc. and M.Sc. in Electronics Engineering from Istanbul Technical University in 1982, 1985, respectively. He has completed his $\mathrm{PhD}$ on $4 \mathrm{G}$ and $5 \mathrm{G}$ radio power frequency amplifiers in 2016 in Istanbul Technical University. He is now working in Netas R\&D. He has more than 20 scientific papers on $5 \mathrm{G}$ communication systems. His research interests include $5 \mathrm{G}$ communication systems, theoretical and practical aspects of radio frequency power amplifier designs. 


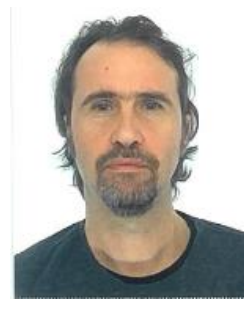

TUĞRUL AKYÜZ has received his B.Sc. in Electronics Engineering from İstanbul Technical University (ITÜ) in 1992 and M.Sc. in Electronics and Telecommunication Engineering from Boğaziçi University (BÜ) in 1996. He is engineer since 1996. 